\title{
Acetone Detection by Chemical Sensors Based on Tungsten and Titanium Oxide Nanowires ${ }^{\dagger}$
}

\author{
Angela Bertuna *, Elisabetta Comini, Nicola Poli, Dario Zappa and Giorgio Sberveglieri \\ SENSOR Laboratory University of Brescia and CNR-INO, Via D. Valotti 9, 25133 Brescia, Italy; \\ elisabetta.comini@unibs.it (E.C.); nicola.poli@unibs.it (N.P.); dario.zappa@unibs.it (D.Z.); \\ giorgio.sberveglieri@unibs.it (G.S.) \\ * Correspondence: a.bertuna@unibs.it; Tel.: +39-030-371-5873 \\ + Presented at the Eurosensors 2017 Conference, Paris, France, 3-6 September 2017.
}

Published: 21 August 2017

\begin{abstract}
Tungsten and titanium oxide $\left(\mathrm{WO}_{3} / \mathrm{Ti}\right)$ nanowires $(\mathrm{NWs})$ were grown by thermal oxidation of a tungsten and titanium alloy (WTi) metallic layer deposited on $2 \times 2 \mathrm{~mm}^{2}$ alumina substrates by DC magnetron sputtering. Thermal oxidation was carried out in a custom evaporator chamber. Several parameters were controlled to achieve the optimal growth conditions. Morphological and structural analysis were performed on samples by a scanning electron microscope (SEM) and RAMAN spectroscopy, respectively. Nanowires, grown directly on the final transducer, were tested towards different gaseous species in a wide range of working temperatures. In this work, results obtained in presence of acetone are proposed.
\end{abstract}

Keywords: acetone; nanowires; tungsten; titanium; chemical sensor

\section{Introduction}

Nowadays volatile organic compounds (VOCs) are intensely studied because of short- and long-term adverse health effects that they can cause. VOCs are the fundamental ingredients for preparation of paints, air fresheners, cosmetics and other products [1]. One of the most used is acetone, a colourless and flammable liquid. It is used in pharmaceutics, and also to purify paraffin, dissolve plastic, and dehydrate tissues. When inhaled, acetone can cause headache, tiredness and even culminate in narcosis and harmfulness to the nervous system [2]. As well as to ensure security in the environments, acetone monitoring is very important in the medical field. People suffering from diabetes have a breath characterized by a fruity scent odour due to the presence of acetone $[2,3]$. An easy detection of this compound could improve diagnosis of the disease, introducing a not-invasive technique of analysis in comparison with current ones. In fact, nowadays diabetes is typically detected starting from blood analysis of patients [3].

In this work, tungsten and titanium oxide nanowires were grown by thermal oxidation of a thin metallic layer in order to build a device that is able to detect acetone.

\section{Materials and Methods}

The starting point of this research is the preparation of the samples. It consists of two steps: the cleaning of the substrates and the deposition of the WTi metallic layer. The first one is necessary to remove organic compounds and particles that can affect all other steps; the second one represents the deposition of the source material used for the growth of nanowires. Alumina substrates $\left(2 \times 2 \mathrm{~mm}^{2}\right)$ were cleaned with acetone in an ultrasonic bath, and then dried under a synthetic airflow. DC Magnetron sputtering was used to deposit the metallic film $(100 \mathrm{~nm})$ on the substrates, starting from a metallic alloy target of tungsten and titanium (W/Ti 90/10 wt \%). 
The technique selected for the growth of metal oxide nanowires was the thermal oxidation and it was carried out in a custom evaporator chamber. The control of various parameters, including pressure, temperature and atmosphere composition inside the chamber, resulted in the achievement of optimum growth conditions. The optimal morphology was reached treating samples at $600{ }^{\circ} \mathrm{C}$, for $1 \mathrm{~h}$ under a flow of $50 \mathrm{sccm}$ of $\mathrm{O}_{2}$. The pressure was set at $1 \times 10^{-1} \mathrm{mbar}$ and it was maintained thanks to the presence of two vacuum pumps: a rotary one and a turbo one.

The presence of $\mathrm{WO}_{3} / \mathrm{Ti}$ nanowires on the surface of the samples was confirmed by morphology analysis carried out by a field effect scanning electron microscope (FE-SEM LEO 1525), by setting the beam voltage at $7 \mathrm{kV}$.

Raman spectroscopy was performed on these samples in order to understand the influence of titanium. The system used for this investigation is composed by:

- Peltier-cooled Synapse

- CCD

- HORIBA monochromator iHR320

- $\quad$ Fiber-coupled confocal optical microscope (HORIBA)

- He-Cd laser (442 nm)

The ability of $\mathrm{WO}_{3} / \mathrm{Ti}$ nanowires to recognize different gaseous species was evaluated in term of resistance/conductance variation due to the interaction between the sensing layer and the gas molecules. In order to do that, platinum interdigitated contacts were deposited directly on the nanowires by DC Magnetron sputtering. The same technique was used to deposit a platinum heater on the other side of the substrate with the aim of working in a wide range of operating temperatures. The assembly of each device was done mounting the samples on TO cases by means of electro-soldered gold wires. Gas sensing measurements were performed in a homemade test chamber, testing, at the same time, 8 sensors powered at $1 \mathrm{~V}$. Several concentrations of different gases were injected in the test chamber. The relative humidity was set to $50 \%$ during all the measurements and the chamber temperature was kept at $20^{\circ} \mathrm{C}$.

\section{Results and Discussion}

Thermal oxidation resulted in a uniform growth of nanowires with a length around $600 \mathrm{~nm}$ and a diameter of $50 \mathrm{~nm}$. Figure 1 shows the morphology of $\mathrm{WO}_{3} / \mathrm{Ti}$ nanowires at two different level magnification.

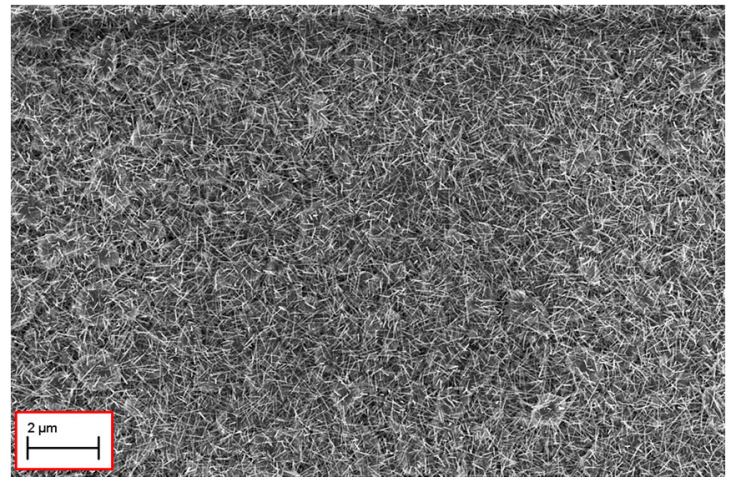

(a)

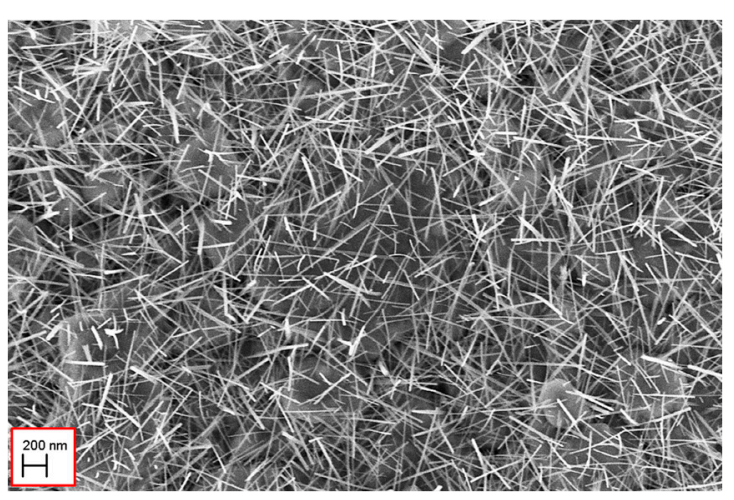

(b)

Figure 1. (a) SEM images of $\mathrm{WO}_{3} / \mathrm{Ti}$ nanowires at low level magnification; (b) SEM images of $\mathrm{WO}_{3} / \mathrm{Ti}$ nanowires at high level magnification.

Raman investigation highlighted the similarity between the spectrum of $\mathrm{WO}_{3} / \mathrm{Ti}$ and the one of $\mathrm{WO}_{3}$. The results of this analysis are shown in Figure 2 where it is possible to see that the main peaks are broadened maybe for the influence of titanium. 


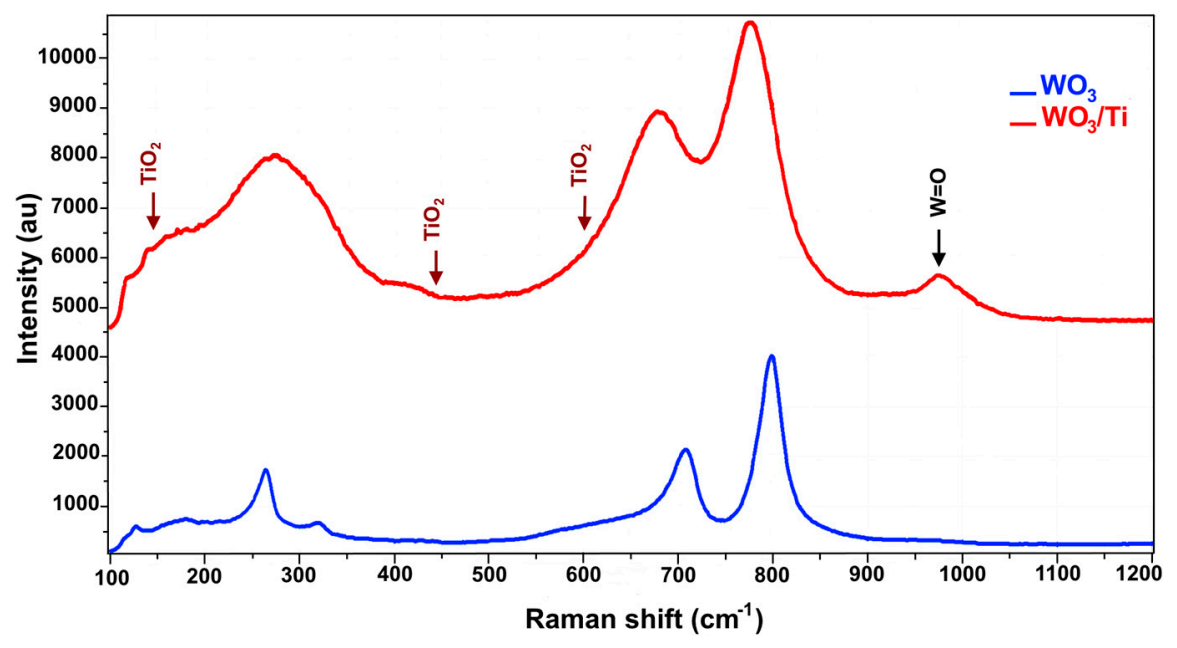

Figure 2. Raman spectrum of $\mathrm{WO}_{3} / \mathrm{Ti}$ (red) in comparison with $\mathrm{WO}_{3}$ (blue) spectrum.

After morphological and structural investigation, several measurements were performed in order to check the ability of chemical sensors based on $\mathrm{WO}_{3} / \mathrm{Ti}$ nanowires in harmful gases detection. Experiments towards many gaseous species were carried out measuring three different concentration for each gas. The operative temperature of each device was controlled in a range between $200{ }^{\circ} \mathrm{C}$ and $550{ }^{\circ} \mathrm{C}$. During all the measurements, the relative humidity was set at $50 \%$ @ $20^{\circ} \mathrm{C}$. This work focuses on the acetone sensing performances. For this reason, the response versus temperature (a), the calibration curve at $500{ }^{\circ} \mathrm{C}(\mathrm{b})$ and dynamic response at the same working temperature (c) towards acetone are shown in Figure 3.
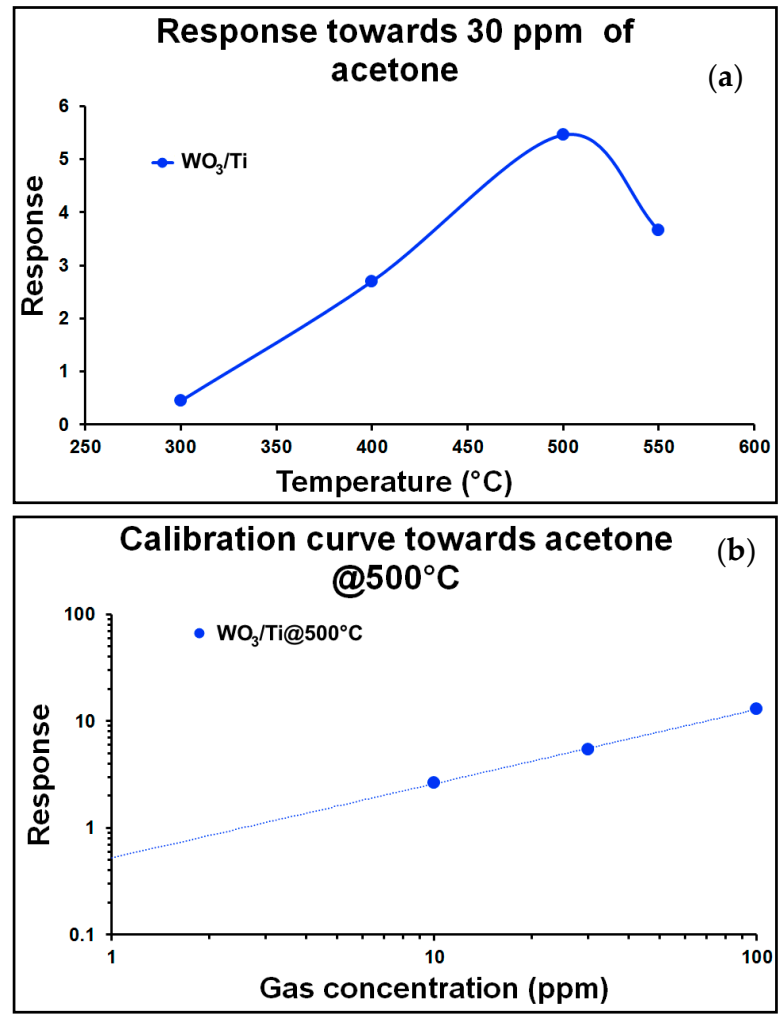


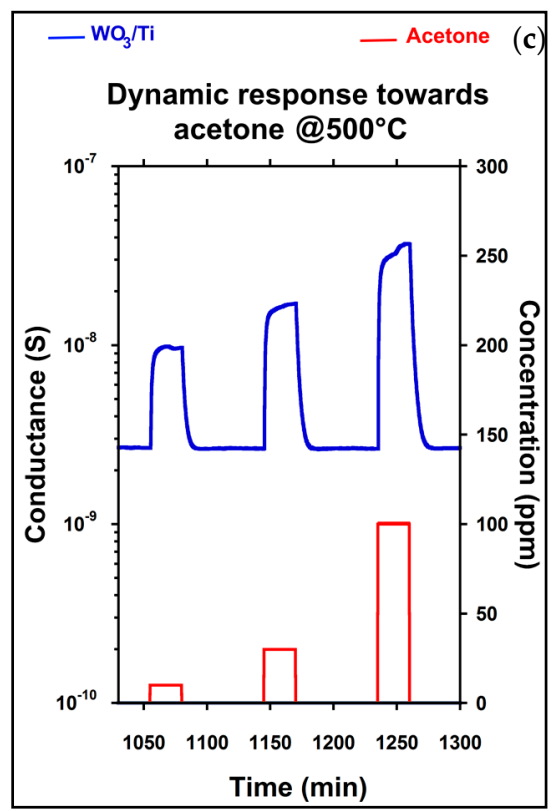

Figure 3. (a) Sensor response versus temperature towards $30 \mathrm{ppm}$ of acetone; (b) Calibration curve at $500{ }^{\circ} \mathrm{C}$ towards different concentration of acetone; (c) Dynamic response at $500{ }^{\circ} \mathrm{C}$ towards acetone.

The detection limit, evaluated considering as minimum response value 1, is around $2.54 \mathrm{ppm}$. Usually, the breath of healthy people contains acetone in a range of 0.3 to $0.9 \mathrm{ppm}$, while in the case of people with diabetes, the acetone concentration rises to over $1.8 \mathrm{ppm}[2,3]$. The limit considered in this paper is very conservative and the possibility of using lower values is not excluded. For example, considering a response of 0.5 , the value of the detection limit drops to $0.93 \mathrm{ppm}$, which is in line with diabetes specifics.

\section{Conclusions}

In this work, $\mathrm{WO}_{3} / \mathrm{Ti}$ nanowires were grown by thermal oxidation technique. Morphological, structural and functional investigation were carried out on these samples. Gas sensing measurements underlines the ability of these devices to recognize different gases. Particular attention was given to acetone, in order to fabricate devices that can be integrated in medical tools for diabetes detection, avoiding invasive techniques currently in use.

Acknowledgments: This work was supported by the European Community's 7th Framework Programme, under the grant agreement n 611887 “MSP: Multi Sensor Platform for Smart Building Management”.

Conflicts of Interest: The authors declare no conflict of interest.

\section{References}

1. Mallya, A.; Kottokkaran, R.; Ramamurthy, P. Conducting polymer-carbon black nanocomposite sensor for volatileorganic compounds and correlating sensor response by moleculardynamics. Sens. Actuators $B$ Chem. 2014, 201, 308-320, doi:10.1016/j.snb.2014.04.056.

2. Song, P.; Wang, Q.; Yang, Z. Preparation, characterization and acetone sensing properties of Ce-doped SnO2. Sens. Actuators B Chem. 2012, 173, 839-846, doi:10.1016/j.snb.2012.07.115.

3. Nasution, T.I.; Nainggolan, I.; Hutagalung, S.; Ahmad, K.; Ahmad, Z. The sensing mechanism and detection of low concentration acetone using chitosan-based sensors. Sens. Actuators B Chem. 2013, 177, 522-528. doi:10.1016/j.snb.2012.11.063.

(C) 2017 by the authors. Licensee MDPI, Basel, Switzerland. This article is an open access article distributed under the terms and conditions of the Creative Commons Attribution (CC BY) license (http://creativecommons.org/licenses/by/4.0/). 